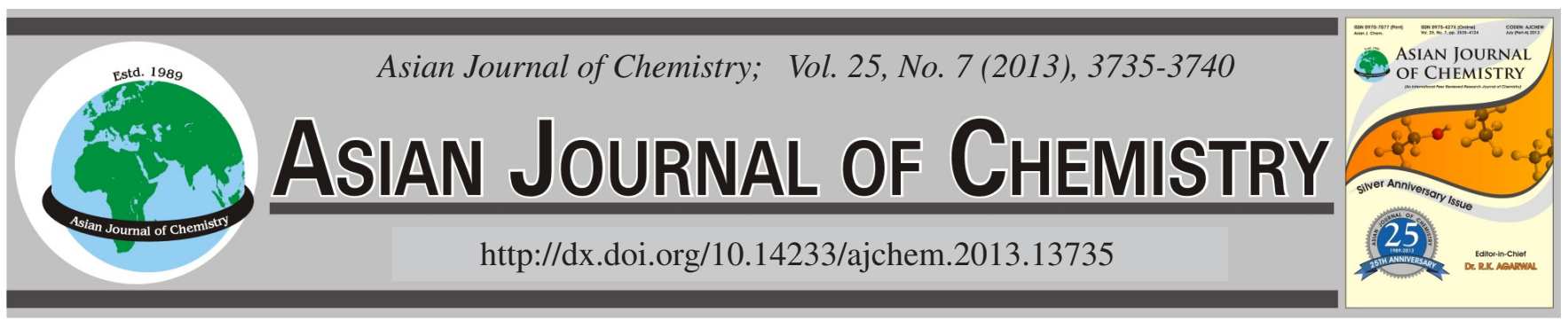

\title{
Synthesis, Characterization and Biological Screening of $\mathrm{N}$-Substituted (5-Chloro-2-methoxyphenyl)benzene Sulfonamide
}

\author{
Aziz-ur-Rehman ${ }^{1, *}$, Ambreen Fatima $^{1}$, Muhammad Athar Abbasi $^{1}$, Khalid Mohammed Khan $^{2}$, \\ Muhammad Ashraf ${ }^{3}$, Irshad Ahmad $^{4}$ and Syeda Abida EJaz ${ }^{4}$
}

\begin{abstract}
${ }^{1}$ Department of Chemistry, Government College University, Lahore-54000, Pakistan
${ }^{2}$ HEJ Research Institute of Chemistry, International Center for Chemical and Biological Sciences, University of Karachi, Karachi-75270, Pakistan ${ }^{3}$ Department of Biochemistry and Biotechnology, The Islamia University of Bahawalpur, Bahawalpur-63100, Pakistan

${ }^{4}$ Department of Pharmacy, The Islamia University of Bahawalpur, Bahawalpur-63100, Pakistan
\end{abstract}

*Corresponding author: Tel: +92 42 111000010; Ext. 449; E-mail: azizryk@yahoo.com

\begin{abstract}
In the present study, a series of $N$-substituted (5-chloro-2-methoxyphenyl)benzene sulfonamide have been synthesized. The reaction of benzene sulfonyl chloride (1) with 2-amino-4-chloroanisole (2) yielded $N$-(5-chloro-2-methoxyphenyl)benzene sulfonamide (3). Finally the target compounds (5a-k) were obtained by stirring $N$-(5-chloro-2-methoxyphenyl)benzene sulfonamide with different electrophiles (4a-k) in the presence of $N, N$-dimethyl formamide and sodium hydride. The structures of the synthesized compounds were established by spectroscopic techniques like ${ }^{1} \mathrm{H}$ NMR and EI-MS. These compounds were assayed for their antioxidant activities by using 2,2-diphenyl1-picrylhydrazil (DPPH) scavenging and other biological activities via screening them against acetylcholinesterase, butyrylcholinesterase and lipoxygenase enzymes, however, these showed prominent activity against acetylcholinesterase enzyme. It is clearly evident from the results that the compounds $N$-methyl-(5-chloro-2-methoxyphenyl)benzene sulfonamide (5a), $N$-allyl- $N$-(5-chloro-2-methoxyphenyl)benzene sulfonamide (5e) and $N$-2"-phenylethyl- $N$-(5-chloro-2-methoxyphenyl)benzene sulfonamide $(\mathbf{5 j})$ were found to be promising inhibitors against acetylcholinesterase enzyme having $\mathrm{IC}_{50}$ value of $34.61 \pm 0.62,40.21 \pm 0.25$ and $45.11 \pm 0.22 \mu \mathrm{mol} / \mathrm{L}$, respectively, relative to Eserine, a reference standard with $\mathrm{IC}_{50}$ value of $0.04 \pm 0.001 \mu \mathrm{mol} / \mathrm{L}$.
\end{abstract}

Key Words: 2-Amino-4-chloroanisole, Benzenesulfonyl chloride, Sulfonamides, Acetylcholinesterase enzyme.

\section{INTRODUCTION}

The sulfonamides belong to class of compounds that comprise at least five different classes of pharmacologically active agents ${ }^{1}$. The basic sulfonamide group $-\mathrm{SO}_{2} \mathrm{NH}$ - occurs in various biological dynamic compounds including antimicrobial drugs, antithyroid agents, antitumor, antibiotics and inhibitors of carbonic anhydrase ${ }^{2}$. Sulfonamides are widely used to treat microbial infections by inhibiting the growth of gram negative and gram positive bacteria, some protozoa and fungi ${ }^{3}$. Clinically sulfonamides are used to treat several urinary tract infections and gastrointestinal infections ${ }^{4}$. Sulfonamides that are aromatic or hetroaromatic are responsible for the inhibition of the growth of tumor cells. They act as antitumor agents by inhibiting the carbonic anhydrase. Sulfonamides are structurally similar to $p$-aminobenzoic acid (PABA) which is a cofactor that is needed by the bacteria for the synthesis of folic acid. Sulfonamides antibiotics inhibit the conversion of $p$-aminobenzoic acid into folic acid and thus ultimately inhibit the synthesis of purine and DNA. Sulfonamide antibiotics are used as veterinary medicines to treat infections in livestock herds ${ }^{5,6}$.

Acetyl cholinesterase (AChE, EC 3.1.1.7) and butyryl cholinesterase (BChE, EC 3.1.1.8) comprise a family of enzymes which include serine hydrolases. The different specificities for substrates and inhibitors for these enzymes are due to the differences in amino acid residues of the active sites of $\mathrm{AChE}$ and BChE. The enzyme system is responsible for the termination of acetylcholine at cholinergic synapses. These are key components of cholinergic brain synapses and neuromuscular junctions. The major function of $\mathrm{AChE}$ and $\mathrm{BChE}$ is to catalyze the hydrolysis of the neurotransmitter acetylcholine and termination of the nerve impulse in cholinergic synapses ${ }^{7}$. It has been found that $\mathrm{BChE}$ is present in significantly higher quantities in Alzheimer's plaques than in the normal age related non dementia of brains. $\mathrm{H}_{1}$ and $\mathrm{H}_{2}$ receptor antagonists possess AChE inhibitory activities. Cholinesterase inhibitors increase the amount of acetylcholine available for neuronal and neuromuscular transmission through their ability to reversibly or 
irreversibly. Hence, the search for new cholinesterase inhibitors is considered an important and ongoing strategy to introduce new drug candidates for the treatment of Alzheimer's disease and other related diseases ${ }^{8-10}$.

Literature survey revealed that slight modification in the structure of sulfonamide can lead to quantitative as well as qualitative changes in the biological activity. It prompted us to synthesize the various $N$-substituted derivatives of sulfonamides with an objective to search new contenders of drug having significant enhanced activity and can be helpful in controlling many degenerative diseases. For this, the parent sulfonamide $N$-(5-chloro-2-methoxyphenyl)benzene sulfonamide (3), was first prepared by reacting benzene sulfonyl chloride with 2-amino-4-chloroanisole at room temperature in basic medium. Simple stirring gave the desired compound in excellent yield. Then it was further processed to obtain different new $\mathrm{N}$-alkyl substituted sulfonamides.

\section{EXPERIMENTAL}

${ }^{1} \mathrm{H}$ NMR spectra were recorded in $\mathrm{CD}_{3} \mathrm{OD}$ on a Burker Aspect AM-500 MHz using TMS as an internal standard. Chemical shifts are given in ppm. IR spectra were recorded in $\mathrm{KBr}$ on a Shimadzu IR-460 and Burker vector 22 spectrometer (wave number in $\mathrm{cm}^{-1}$ ). Mass spectra (EIMS) were measured on Finnigan MAT-112 instrument. High resolution EIMS were recorded in Jeol JMS HX-110 spectrometer. The melting points were recorded on a Griffin \& George melting point apparatus. TLC was conducted on $0.25 \mathrm{~mm}$ silica gel plates. Visualization was made with ultraviolet light.

Procedure for the synthesis of $N$-(5-chloro-2-methoxyphenyl)benzene sulfonamide (3): The nucleophilic substitution reaction of amine with aryl sulfonyl chloride was carried out in aqueous media as described in the literature ${ }^{11}$ : a mixture 2-amino-4-chloroanisole (1; $10.0 \mathrm{mmol} ; 1.47 \mathrm{~g})$ and benzene sulfonyl chloride $(2 ; 10.0 \mathrm{mmol} ; 1.45 \mathrm{~mL})$ and was suspended in $25 \mathrm{~mL}$ water. The $\mathrm{pH}$ of the suspension was maintained at 9.0 by adding aqueous solution of $\mathrm{Na}_{2} \mathrm{CO}_{3}$ at room temperature. The mixture was stirred and monitored by using analytical technique TLC for the completion of reaction. Then concentrated $\mathrm{HCl}$ was added gradually to adjust the $\mathrm{pH}$ to 2.0. The precipitates were collected by filtration, washed with distilled $\mathrm{H}_{2} \mathrm{O}$ and dried to afford the title compound 3. Methyl alcohol was used to dissolve the product and then it was re-crystallized by slow evaporation of the solvent, to generate colourless needle like crystals of $N$-(5-chloro-2-methoxyphenyl)benzene sulfonamide.

General method for the synthesis of compounds (5ak): The calculated amount of compound $3(0.1 \mathrm{mmol})$ was taken in a round bottomed flask $(50 \mathrm{~mL})$ and $N, N$-dimethyl formamide $(10 \mathrm{~mL})$ was added to dissolve it followed by the addition of sodium hydride/lithium hydride $(0.1 \mathrm{mmol})$ to the reaction mixture. The mixture was stirred for $0.5 \mathrm{~h}$ at ambient temperature and then slowly added the electrophile to the mixture and was further stirred for $2 \mathrm{~h}$. The progress of reaction was monitored via TLC. The product was precipitated by adding cold distilled water. It was filtered, washed with water and crystallized from aqueous methanol.
Acetylcholinesterase assay: The AChE inhibition activity was performed according to the reported method ${ }^{12}$ with slight modifications. Total volume of the reaction mixture was 100 $\mu \mathrm{L}$. It contained $60 \mu \mathrm{L} \mathrm{Na}_{2} \mathrm{HPO}_{4}$ buffer with concentration of $50 \mathrm{mM}$ and $\mathrm{pH}$ 7.7. Ten $\mu \mathrm{L}$ test compound $\left(0.5 \mathrm{mM} \mathrm{well}^{-1}\right)$ was added, followed by the addition of $10 \mu \mathrm{L}\left(0.005\right.$ unit well $\left.^{-1}\right)$ enzyme. The contents were mixed and pre-read at $405 \mathrm{~nm}$. Then contents were pre-incubated for $10 \mathrm{~min}$ at $37^{\circ} \mathrm{C}$. The reaction was initiated by the addition of $10 \mu \mathrm{L}$ of $0.5 \mathrm{mM}$ well $^{-1}$ substrate (acetylthiocholine iodide), followed by the addition of $10 \mu \mathrm{L}$ DTNB $\left(0.5 \mathrm{mM}\right.$ well $\left.{ }^{-1}\right)$. After $15 \mathrm{~min}$ of incubation at $37^{\circ} \mathrm{C}$ absorbance was measured at $405 \mathrm{~nm}$ using 96-well plate reader Synergy HT, Biotek, USA. All experiments were carried out with their respective controls in triplicate. Eserine $\left(0.5 \mathrm{mM}\right.$ well $\left.{ }^{-1}\right)$ was used as a positive control. The percent inhibition was calculated by the help of following equation

$$
\text { Inhibition }(\%)=\frac{\text { Control }- \text { Test }}{\text { Control }} \times 100
$$

$\mathrm{IC}_{50}$ values were calculated using EZ-Fit Enzyme kinetics software (Perrella Scientific Inc. Amherst, USA).

Butyrylcholinesterase assay: The $\mathrm{BChE}$ inhibition activity was performed according to the reported method ${ }^{12}$ with slight modifications. Total volume of the reaction mixture was $100 \mu \mathrm{L}$ containing $60 \mu \mathrm{L}, \mathrm{Na}_{2} \mathrm{HPO}_{4}$ buffer, $50 \mathrm{mM}$ and $\mathrm{pH}$ 7.7. Ten $\mu \mathrm{L}$ test compound $0.5 \mathrm{mM}$ well ${ }^{-1}$ was added followed by the addition of $10 \mu \mathrm{L}\left(0.5\right.$ unit well $\left.^{-1}\right) \mathrm{BChE}$ (Sigma Inc.). The contents were mixed and pre-read at $405 \mathrm{~nm}$ and then pre-incubated for $10 \mathrm{~min}$ at $37^{\circ} \mathrm{C}$. The reaction was initiated by the addition of $10 \mu \mathrm{L}$ of $0.5 \mathrm{mM}$ well ${ }^{-1}$ substrate (butyrylthiocholine chloride). Followed by the addition of $10 \mu \mathrm{L}$ DTNB, $0.5 \mathrm{mM}$ well ${ }^{-1}$. After $15 \mathrm{~min}$ of incubation at $37^{\circ} \mathrm{C}$, absorbance was measured at $405 \mathrm{~nm}$ using 96-well plate reader Synergy HT, Biotek, USA. All experiments were carried out with their respective controls in triplicate. Eserine $(0.5 \mathrm{mM}$ well $^{-1}$ ) was used as positive control. The percent inhibition was calculated by the help of following equation.

$$
\text { Inhibition }(\%)=\frac{\text { Control }- \text { Test }}{\text { Control }} \times 100
$$

$\mathrm{IC}_{50}$ values were calculated using EZ-Fit Enzyme kinetics software (Perrella Scientific Inc. Amherst, USA).

Lipoxygenase assay: Lipoxygenase (LOX) activity was assayed according to the reported method ${ }^{13-15}$ with slight modifications. A total volume of $200 \mu \mathrm{L}$ lipoxygenase assay mixture contained $150 \mu \mathrm{L}$ sodium phosphate buffer (100 mM, pH 8.0), $10 \mu \mathrm{L}$ test compound and $15 \mu \mathrm{L}$ purified lipoxygenase enzyme (600 units well ${ }^{-1}$, Sigma Inc.). The contents were mixed and pre-read at $234 \mathrm{~nm}$ and preincubated for $10 \mathrm{~min}$ at $25^{\circ} \mathrm{C}$. The reaction was initiated by addition of $25 \mu \mathrm{L}$ substrate solution. The change in absorbance was observed after 6 min at 234 nm using 96-well plate reader Synergy HT, Biotek, USA. All reactions were performed in triplicates. The positive and negative controls were included in the assay. Baicalin $\left(0.5 \mathrm{mM} \mathrm{well}^{-1}\right)$ was used as a positive control. The percentage inhibition (\%) was calculated by formula given below.

$$
\text { Inhibition }(\%)=\frac{\text { Control }- \text { Test }}{\text { Control }} \times 100
$$


where, Control $=$ Total enzyme activity without inhibitor. Test $=$ Activity in the presence of test compound. $\mathrm{IC}_{50}$ values were calculated using EZ-Fit Enzyme Kinetics software (Perrella Scientific Inc. Amherst, USA).

DPPH assay: The stable 1,1-diphenyl-2-picrylhydrazyl radical (DPPH) was used for the determination of antioxidant activity. Different concentrations of compounds in respective solvents were added at an equal volume $(10 \mu \mathrm{L})$ to $90 \mu \mathrm{L}$ of $100 \mu \mathrm{M}$ methanolic DPPH in a total volume of $100 \mu \mathrm{L}$ in 96-well plates. The contents were mixed and incubated at $37^{\circ} \mathrm{C}$ for $0.5 \mathrm{~h}$. The absorbance was measured at $517 \mathrm{~nm}$ using Synergy HT BioTek® USA microplate reader. Quercetin and L-ascorbic acid were used as standard antioxidants. The experiments were carried out in triplicates. $\mathrm{IC}_{50}$ values were calculated using EZ-Fit5 Perrella Scientific Inc. Amherst USA software. The decrease in absorbance indicates increased radical scavenging activity which was determined by the following formula ${ }^{16}$.

$$
\text { Antiradical activity }(\text { Inhibition } \%)=\frac{\text { Control }- \text { Test }}{\text { Control }} \times 100
$$

Statistical analysis: All the measurements were done in triplicate and statistical analysis was performed by Microsoft Excel 2003. Results are presented as mean \pm sem.

\section{Spectral characterization of the synthesized compounds}

$N$-(5-Chloro-2-methoxyphenyl)benzene sulfonamide (3): Colourless needle like crystals, Yield: $91 \%$, m.p. 140$142{ }^{\circ} \mathrm{C}$. IR (KBr, $\left.v_{\max }, \mathrm{cm}^{-1}\right): 3120$ ( $N$-H str.), $3020(\mathrm{C}-\mathrm{H}$ aromatic str.), 1447, $1338\left(\mathrm{SO}_{2}\right), 1225\left(-\mathrm{OCH}_{3}\right) ;{ }^{1} \mathrm{H}$ NMR $(500$ $\left.\mathrm{MHz}, \mathrm{CD}_{3} \mathrm{OD}\right): \delta 7.71\left(\mathrm{dd}, J=7.5,1.5 \mathrm{~Hz}, 2 \mathrm{H}, \mathrm{H}-2^{\prime}\right.$ and H-6'), 7.55 (br.t, $J=7.5 \mathrm{~Hz}, 1 \mathrm{H}, \mathrm{H}-4^{\prime}$ ), 7.45 (br.t, $J=7.5 \mathrm{~Hz}$, 2H, H-3' and H-5'), 7.41 (d, $J=2.5 \mathrm{~Hz}, 1 \mathrm{H}, \mathrm{H}-6), 7.05$ (dd, $J=8.5,2.5 \mathrm{~Hz}, 1 \mathrm{H}, \mathrm{H}-4), 6.79$ (d, $J=8.5 \mathrm{~Hz}, 1 \mathrm{H}, \mathrm{H}-3), 3.51$ (s, 3H, - $\left.\mathrm{OCH}_{3}\right)$; EIMS: m/z $297\left[\mathrm{M}^{+}\right], 288\left[\mathrm{M}-\mathrm{CH}_{3}\right]^{+}, 266$ $\left[\mathrm{M}-\mathrm{OCH}_{3}\right]^{+}, 233\left[\mathrm{M}-\mathrm{SO}_{2}\right]^{+}, 156\left[\mathrm{M}-\mathrm{PhSO}_{2}\right]^{+}, 141[\mathrm{M}-$ $\left.\mathrm{C}_{6} \mathrm{H}_{3}(\mathrm{Cl})\left(\mathrm{OCH}_{3} \mathrm{NH}\right)\right]^{+}$.

$\mathrm{N}$-Methyl-(5-chloro-2-methoxyphenyl)benzene sulfon amide (5a): Yellow coloured crystals, yield: $83 \%$. m.p. 110$112^{\circ} \mathrm{C}$. IR $\left(\mathrm{KBr}, \mathrm{v}_{\max }, \mathrm{cm}^{-1}\right): 3018$ (aromatic C-H), 1445, 1340 $\left.\left(\mathrm{SO}_{2}\right), 1220\left(-\mathrm{OCH}_{3}\right)\right), 1150$ (aliphatic C-N str.); ${ }^{1} \mathrm{H}$ NMR (500 $\left.\mathrm{MHz}, \mathrm{CD}_{3} \mathrm{OD}\right): \delta 7.65\left(\mathrm{dd}, J=7.5,1.5 \mathrm{~Hz}, 2 \mathrm{H}, \mathrm{H}-2^{\prime}\right.$ and $\mathrm{H}-$ 6'), 7.62 (br.t, $J=7.5 \mathrm{~Hz}, 1 \mathrm{H}, \mathrm{H}-4$ '), 7.53 (br.t, $J=7.5 \mathrm{~Hz}, 2 \mathrm{H}$, H-3' and H-5'), 7.29 (dd, $J=8.5,2.5 \mathrm{~Hz}, 1 \mathrm{H}, \mathrm{H}-4), 7.23$ (d, $J=$ $2.5 \mathrm{~Hz}, 1 \mathrm{H}, \mathrm{H}-6), 6.89$ (d, J = 8.5 Hz, 1H, H-3), 3.34 (s, 3H, $\left.-\mathrm{OCH}_{3}\right), 3.18$ (s, 3H, -CH $\mathrm{CH}_{3}$; EIMS: m/z $311\left[\mathrm{M}^{+}\right], 296$ [M$\left.\mathrm{CH}_{3}\right]^{+}, 280\left[\mathrm{M}-\mathrm{OCH}_{3}\right]^{+}, 247\left[\mathrm{M}-\mathrm{SO}_{2}\right]^{+}, 170\left[\mathrm{M}-\mathrm{PhSO}_{2}\right]^{+}, 141$ $\left[\mathrm{PhSO}_{2}\right]^{+}$.

$N$-Ethyl- $N$-(5-chloro-2-methoxyphenyl)benzene sulfon amide (5b): White amorphous powder, Yield: $79 \%$, m.p. 79$81^{\circ} \mathrm{C}$. IR (KBr, $v_{\max }, \mathrm{cm}^{-1}$ ): 3022 (aromatic C-H), 1444, 1337 $\left(\mathrm{SO}_{2}\right), 1227$ (-OCH$), 1151$ (aliphatic C-N str.); ${ }^{1} \mathrm{H}$ NMR (500 $\mathrm{MHz}, \mathrm{CD}_{3} \mathrm{OD}$ ): $\delta 7.64\left(\mathrm{dd}, J=7.5,1.5 \mathrm{~Hz}, 2 \mathrm{H}, \mathrm{H}-2^{\prime}\right.$ and H-6'), 7.61 (br.t, $J=7.5 \mathrm{~Hz}, 1 \mathrm{H}, \mathrm{H}-4^{\prime}$ ), 7.52 (br.t, $J=7.5 \mathrm{~Hz}$, 2H, H-3' and H-5'), 7.32 (dd, $J=8.5,2.5 \mathrm{~Hz}, 1 \mathrm{H}, \mathrm{H}-4), 7.19$ $(\mathrm{d}, J=2.5 \mathrm{~Hz}, 1 \mathrm{H}, \mathrm{H}-6), 6.80$ (d, $J=8.5 \mathrm{~Hz}, 1 \mathrm{H}, \mathrm{H}-3), 3.63$ (br.s, 2H, $\mathrm{CH}_{2}-1^{\prime \prime}$ ), 3.34 (s, 3H,-OCH $), 1.03$ (t, $J=7.5 \mathrm{~Hz}$, $\left.3 \mathrm{H}, \mathrm{CH}_{3}-2 "\right)$; EIMS: m/z $325\left[\mathrm{M}^{+}\right], 310\left[\mathrm{M}-\mathrm{CH}_{3}\right]^{+}, 294$ [M$\left.\mathrm{OCH}_{3}\right]^{+}, 261\left[\mathrm{M}-\mathrm{SO}_{2}\right]^{+}, 184\left[\mathrm{M}-\mathrm{PhSO}_{2}\right]^{+}, 141\left[\mathrm{PhSO}_{2}\right]^{+}$.
$N$-Isopropyl- $N$-(5-chloro-2-methoxyphenyl)benzene sulfonamide (5c): Brown needle like crystals, Yield: $59 \%$, m.p. $90-92^{\circ} \mathrm{C}$. IR (KBr, $\left.v_{\max }, \mathrm{cm}^{-1}\right)$ : 3019 (aromatic C-H), 1446, $1342\left(\mathrm{SO}_{2}\right), 1229\left(-\mathrm{OCH}_{3}\right), 1149$ (aliphatic C-N str.); ${ }^{1} \mathrm{H} \mathrm{NMR}$ (500 MHz, $\left.\mathrm{CD}_{3} \mathrm{OD}\right): \delta 7.64$ (dd, $J=7.5,1.5 \mathrm{~Hz}, 2 \mathrm{H}, \mathrm{H}-2^{\prime}$ and H-6'), 7.61 (br.t, $J=7.5 \mathrm{~Hz}, 1 \mathrm{H}, \mathrm{H}-4$ '), 7.52 (br.t, $J=7.5 \mathrm{~Hz}$, 2H, H-3' and H-5'), 7.32 (dd, $J=8.5,2.5 \mathrm{~Hz}, 1 \mathrm{H}, \mathrm{H}-4), 7.19$ (d, $J=2.5 \mathrm{~Hz}, 1 \mathrm{H}, \mathrm{H}-6), 6.80$ (d, $J=8.5 \mathrm{~Hz}, 1 \mathrm{H}, \mathrm{H}-3), 4.50$ (m, 1H, H-1"), 3.34 (s, 3H, $\left.-\mathrm{OCH}_{3}\right), 1.01$ (d, $J=6.6 \mathrm{~Hz}, 6 \mathrm{H}$, $\mathrm{CH}_{3}-2$ " and $\mathrm{CH}_{3}-3$ "); EIMS: m/z $339\left[\mathrm{M}^{+}\right], 324\left[\mathrm{M}-\mathrm{CH}_{3}\right]^{+}$, $308\left[\mathrm{M}-\mathrm{OCH}_{3}\right]^{+}, 275\left[\mathrm{M}-\mathrm{SO}_{2}\right]^{+}, 198\left[\mathrm{M}-\mathrm{PhSO}_{2}\right]^{+}, 198$ $\left[\mathrm{PhSO}_{2}\right]^{+}$

$\mathrm{N}$-(2-Bromoethyl)- $\mathrm{N}$-(5-chloro-2-methoxyphenyl)benzene sulfonamide (5d): Dark brown solid, Yield: $64 \%$, IR (KBr, $\left.v_{\max }, \mathrm{cm}^{-1}\right)$ : 3022 (aromatic C-H), 1449, $1337\left(\mathrm{SO}_{2}\right), 1232$ $\left(-\mathrm{OCH}_{3}\right), 1145$ (aliphatic C-N str.); ${ }^{1} \mathrm{H}$ NMR (500 MHz, $\mathrm{CD}_{3} \mathrm{OD}$ ): $\delta 7.96$ (br.s, H-2' and H-6'), 7.62 (m, 1H, H-4'), 7.51 (br.t, $J=7.5 \mathrm{~Hz}, 2 \mathrm{H}, \mathrm{H}-3^{\prime}$ and H-5'), 7.37 (d, $J=2.5 \mathrm{~Hz}, 1 \mathrm{H}$, H-6), 7.32 (dd, $J=8.5,2.5 \mathrm{~Hz}, 1 \mathrm{H}, \mathrm{H}-4), 6.88$ (d, $J=8.5 \mathrm{~Hz}$, 1H, H-3), 3.90 (br.s, 2H, $\mathrm{CH}_{2}-1 "$ ), 3.43 (t, $J=6.5,2 \mathrm{H}, \mathrm{CH}_{2-}$ 2"), 3.27(s, 3H, - $\mathrm{OCH}_{3}$ ); EIMS: m/z $404\left[\mathrm{M}^{+}\right], 389\left[\mathrm{M}-\mathrm{CH}_{3}\right]^{+}$, $373\left[\mathrm{M}-\mathrm{OCH}_{3}\right]^{+}, 340\left[\mathrm{M}-\mathrm{SO}_{2}\right]^{+}, 263\left[\mathrm{M}-\mathrm{PhSO}_{2}\right]^{+}, 141$ $\left[\mathrm{PhSO}_{2}\right]^{+}$.

$\mathrm{N}$-Allyl- $\mathrm{N}$-(5-chloro-2-methoxyphenyl)benzene sulfon amide (5e): Dark brown gammy solid, Yield: $82 \%$, IR (KBr, $\left.v_{\max }, \mathrm{cm}^{-1}\right): 3019$ (aromatic C-H), 1443, $1336\left(\mathrm{SO}_{2}\right), 1227$ $\left(-\mathrm{OCH}_{3}\right), 1151$ (aliphatic C-N str.); ${ }^{1} \mathrm{H}$ NMR $(500 \mathrm{MHz}$, $\mathrm{CD}_{3} \mathrm{OD}$ ): $\delta 7.64$ (dd, $J=7.5,1.5 \mathrm{~Hz}, 2 \mathrm{H}, \mathrm{H}-2^{\prime}$ and $\left.\mathrm{H}-66^{\prime}\right), 7.62$ (br.t, $J=7.5 \mathrm{~Hz}, 1 \mathrm{H}, \mathrm{H}-4^{\prime}$ ) , 7.51 (br.t, $J=7.5 \mathrm{~Hz}, 2 \mathrm{H}, \mathrm{H}-3^{\prime}$ and $\left.\mathrm{H}^{\prime} 5^{\prime}\right), 7.27$ (dd, $\left.J=8.5,2.5 \mathrm{~Hz}, 1 \mathrm{H}, \mathrm{H}-4\right), 7.17$ (d, $J=2.5$ $\mathrm{Hz}, 1 \mathrm{H}, \mathrm{H}-6), 6.85$ (d, J = 8.5 Hz, 1H, H-3), 5.74 (m, 1H, H2"), 5.06 (dd, $\left.J=17.5,1.5 \mathrm{~Hz}, 1 \mathrm{H}, \mathrm{H}_{\mathrm{b}}-3 "\right), 5.00$ (dd, $J=10$, $\left.1.0 \mathrm{~Hz}, 1 \mathrm{H}, \mathrm{H}_{\mathrm{a}}-3^{\prime \prime}\right), 4.20$ (br.s, $\left.2 \mathrm{H}, \mathrm{H}-1 "\right), 3.33$ (s, 3H, $-\mathrm{OCH}_{3}$ ); EIMS: m/z $337\left[\mathrm{M}^{+}\right], 322\left[\mathrm{M}-\mathrm{CH}_{3}\right]^{+}, 306\left[\mathrm{M}-\mathrm{OCH}_{3}\right]^{+}, 273$ $\left[\mathrm{M}-\mathrm{SO}_{2}\right]^{+}, 183\left[\mathrm{M}-\mathrm{PhSO}_{2}\right]^{+}, 141\left[\mathrm{M}-\mathrm{C}_{6} \mathrm{H}_{3}(\mathrm{Cl})\left(\mathrm{OCH}_{3}\right)\right.$ $\left.\mathrm{NCH}_{2} \mathrm{CH}=\mathrm{CH}_{2}\right]^{+}$.

$\mathrm{N}$-Butyl- $\mathrm{N}$-(5-chloro-2-methoxyphenyl)benzene sulfon amide (5f): Colourless sticky solid, Yield: $72 \%$, IR (KBr, $v_{\max }$, $\left.\mathrm{cm}^{-1}\right)$ : 3015 (aromatic C-H), 1445, $1337\left(\mathrm{SO}_{2}\right), 1225\left(-\mathrm{OCH}_{3}\right)$, 1150 (aliphatic C-N str.); ${ }^{1} \mathrm{H}$ NMR (500 MHz, $\left.\mathrm{CD}_{3} \mathrm{OD}\right): \delta$ 7.62 (dd, $J=7.5,1.5 \mathrm{~Hz}, 2 \mathrm{H}, \mathrm{H}-2^{\prime}$ and H-6'), 7.60 (br.t, $J=7.5$ Hz, 1H, H-4'), 7.51 (br.t, $J=7.5 \mathrm{~Hz}, 2 \mathrm{H}, \mathrm{H}-3^{\prime}$ and $\left.\mathrm{H}-5^{\prime}\right), 7.30$ (dd, $J=8.5,2.5 \mathrm{~Hz}, 1 \mathrm{H}, \mathrm{H}-4), 7.18$ (d, $J=2.5 \mathrm{~Hz}, 1 \mathrm{H}, \mathrm{H}-6)$, 6.88 (d, $J=8.5 \mathrm{~Hz}, 1 \mathrm{H}, \mathrm{H}-3$ ), 3.57 (br.s, 2H, H-1"), 3.33 (s, $3 \mathrm{H},-\mathrm{OCH}_{3}$ ), 1.38 (m, 2H, H-3"), 1.27 (m, 2H, H-2"), 0.85 (t, $J=7.0 \mathrm{~Hz}, 3 \mathrm{H}, \mathrm{H}-4 ")$; EIMS: m/z $353\left[\mathrm{M}^{+}\right], 338\left[\mathrm{M}_{-} \mathrm{CH}_{3}\right]^{+}$, $322\left[\mathrm{M}-\mathrm{OCH}_{3}\right]^{+}, 289\left[\mathrm{M}-\mathrm{SO}_{2}\right]^{+}, 212\left[\mathrm{M}-\mathrm{PhSO}_{2}\right]^{+}, 141$ $\left[\mathrm{PhSO}_{2}\right]^{+}$.

$N$-Pentyl- $N$-(5-chloro-2-methoxyphenyl)benzene sulfonamide (5g): Off white solid, Yield: $67 \%$, IR (KBr, $v_{\max }$, $\mathrm{cm}^{-1}$ ): 3016 (aromatic C-H), 1442, $1339\left(\mathrm{SO}_{2}\right), 1224\left(-\mathrm{OCH}_{3}\right)$, 1152 (aliphatic C-N str.); ${ }^{1} \mathrm{H}$ NMR (500 MHz, $\left.\mathrm{CD}_{3} \mathrm{OD}\right): \delta$ 7.62 (dd, $J=7.5,1.5 \mathrm{~Hz}, 2 \mathrm{H}, \mathrm{H}-2^{\prime}$ and H-6'), 7.60 (br.t, $J=7.5$ Hz, 1H, H-4' ), 7.51 (br.t, $J=7.5 \mathrm{~Hz}, 2 \mathrm{H}, \mathrm{H}-3^{\prime}$ and H-5' ), 7.32 (dd, $J=8.5,2.5 \mathrm{~Hz}, 1 \mathrm{H}, \mathrm{H}-4), 7.18$ (d, $J=2.5 \mathrm{~Hz}, 1 \mathrm{H}, \mathrm{H}-6$ ), 6.90 (d, $J=8.5 \mathrm{~Hz}, 1 \mathrm{H}, \mathrm{H}-3$ ), 3.58 (br.s, 2H, H-1"), 3.32 (s, $\left.3 \mathrm{H},-\mathrm{OCH}_{3}\right), 1.36$ (m, 2H, H-2"), 1.33-1.30 (m, 4H, H-3" and H-4"), 0.86 (t, $J=7.5 \mathrm{~Hz}, 3 \mathrm{H}, \mathrm{H}-5 ")$; EIMS: m/z 367 [M+], 
$352\left[\mathrm{M}-\mathrm{CH}_{3}\right]^{+}, 336\left[\mathrm{M}-\mathrm{OCH}_{3}\right]^{+}, 303\left[\mathrm{M}-\mathrm{SO}_{2}\right]^{+}, 226[\mathrm{M}-$ $\left.\mathrm{PhSO}_{2}\right]^{+}, 141\left[\mathrm{PhSO}_{2}\right]^{+}$.

$\mathrm{N}$-Benzyl- $\mathrm{N}$-(5-chloro-2-methoxyphenyl)benzene sulfonamide (5h): Colourless sticky solid, Yield: $75 \%$, IR $\left(\mathrm{KBr}, v_{\max }, \mathrm{cm}^{-1}\right): 3020$ (aromatic C-H), 1447, $1338\left(\mathrm{SO}_{2}\right), 1225$ $\left(-\mathrm{OCH}_{3}\right), 1150$ (aliphatic C-N str.); ${ }^{1} \mathrm{H}$ NMR $(500 \mathrm{MHz}$, $\mathrm{CD}_{3} \mathrm{OD}$ ): $\delta 7.60$ (dd, $J=7.5,1.5 \mathrm{~Hz}, 2 \mathrm{H}, \mathrm{H}-2^{\prime}$ and $\left.\mathrm{H}-6^{\prime}\right), 7.59$ (br.t, $J=7.5 \mathrm{~Hz}, 1 \mathrm{H}, \mathrm{H}-4^{\prime}$ ), 7.48 (br.t, $J=7.5 \mathrm{~Hz}, 2 \mathrm{H}, \mathrm{H}-3^{\prime}$ and $\left.\mathrm{H}^{-5}{ }^{\prime}\right), 7.30(\mathrm{dd}, J=8.5,2.5 \mathrm{~Hz}, 1 \mathrm{H}, \mathrm{H}-4), 7.18(\mathrm{~d}, J=2.5$ $\mathrm{Hz}, 1 \mathrm{H}, \mathrm{H}-6$ ), 7.12-6.95 (m, 5H, H-2" to H-6"), 6.88 (d, $J=$ $8.5 \mathrm{~Hz}, 1 \mathrm{H}, \mathrm{H}-3$ ), 4.67 (s, 2H, - $\mathrm{CH}_{2}$-of benzyl), 3.31 (s, 3H, $\mathrm{OCH}_{3}$ ); EIMS: $\mathrm{m} / \mathrm{z} 387\left[\mathrm{M}^{+}\right], 372\left[\mathrm{M}-\mathrm{CH}_{3}\right]^{+}, 356\left[\mathrm{M}-\mathrm{OCH}_{3}\right]^{+}$,

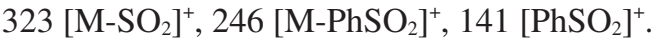

$N$-4"'-Bromobenzyl- $N$-(5-chloro-2-methoxyphenyl)benzene sulfonamide (5i): Brown coloured sticky solid, Yield: $81 \%$, IR ( $\left.\mathrm{KBr}, v_{\max }, \mathrm{cm}^{-1}\right): 3013$ (C-H aromatic str.), 1442, $1334\left(\mathrm{SO}_{2}\right), 1221\left(-\mathrm{OCH}_{3}\right), 1145$ (C-N str.); ${ }^{1} \mathrm{H}$ NMR (500 $\mathrm{MHz}, \mathrm{CD}_{3} \mathrm{OD}$ ): $\delta 7.69$ (d, $J=8.5 \mathrm{~Hz}, 2 \mathrm{H}, \mathrm{H}-3 "$ and $\left.\mathrm{H}-5 "\right)$, $7.63\left(\mathrm{dd}, J=7.5,1.5 \mathrm{~Hz}, 2 \mathrm{H}, \mathrm{H}-2^{\prime}\right.$ and $\left.\mathrm{H}-6^{\prime}\right), 7.61$ (br.t, $J=7.5$ Hz, 1H, H-4'), 7.53 (br.t, $J=7.5 \mathrm{~Hz}, 2 \mathrm{H}, \mathrm{H}-3^{\prime}$ and $\mathrm{H}-5^{\prime}$ ), 7.35 (dd, $J=8.5,2.5 \mathrm{~Hz}, 1 \mathrm{H}, \mathrm{H}-4), 7.28(\mathrm{~d}, J=8.5 \mathrm{~Hz}, 2 \mathrm{H}, \mathrm{H}-2 "$ and H-6"), 7.21 (d, $J=2.5 \mathrm{~Hz}, 1 \mathrm{H}, \mathrm{H}-6), 6.93$ (d, $J=8.5 \mathrm{~Hz}$, $1 \mathrm{H}, \mathrm{H}-3), 4.75$ (s, $2 \mathrm{H},-\mathrm{CH}_{2}$-of benzyl), 3.35 (s, $3 \mathrm{H},-\mathrm{OCH}_{3}$ ); EIMS: m/z $466\left[\mathrm{M}^{+}\right], 451\left[\mathrm{M}-\mathrm{CH}_{3}\right]^{+}, 435\left[\mathrm{M}-\mathrm{OCH}_{3}\right]^{+}, 402$ $\left[\mathrm{M}-\mathrm{SO}_{2}\right]^{+}, 325\left[\mathrm{M}-\mathrm{PhSO}_{2}\right]^{+}, 141\left[\mathrm{PhSO}_{2}\right]^{+}$.

$\mathrm{N}$-2"'-Phenylethyl- $\mathrm{N}$-(5-chloro-2-methoxyphenyl)benzene sulfonamide $(\mathbf{5 j})$ : Colourless sticky solid, Yield: 58 $\%, 67-69^{\circ} \mathrm{C}$. IR $\left(\mathrm{KBr}, v_{\max }, \mathrm{cm}^{-1}\right): 3019$ (aromatic C-H), 1448, $1337\left(\mathrm{SO}_{2}\right), 1223\left(-\mathrm{OCH}_{3}\right), 1154$ (aliphatic C-N str.); ${ }^{1} \mathrm{H}$ NMR (500 MHz, $\left.\mathrm{CD}_{3} \mathrm{OD}\right): \delta 7.61(\mathrm{dd}, J=7.5,1.5 \mathrm{~Hz}, 2 \mathrm{H}, \mathrm{H}-2$ ' and H-6'), 7.58 (br.t, $J=7.5 \mathrm{~Hz}, 1 \mathrm{H}, \mathrm{H}-4^{\prime}$ ) , 7.49 (br.t, $J=7.5 \mathrm{~Hz}$, $2 \mathrm{H}, \mathrm{H}-3^{\prime}$ and $\left.\mathrm{H}^{-} 5^{\prime}\right), 7.30(\mathrm{dd}, J=8.5,2.5 \mathrm{~Hz}, 1 \mathrm{H}, \mathrm{H}-4), 7.19$ (d, $J=2.5 \mathrm{~Hz}, 1 \mathrm{H}, \mathrm{H}-6), 7.12-6.92(\mathrm{~m}, 5 \mathrm{H}, \mathrm{H}-2 "$ to $\mathrm{H}-6 ")$, 6.87 (d, $J=8.5 \mathrm{~Hz}, 1 \mathrm{H}, \mathrm{H}-3$ ), 3.70 (t, $J=7.8 \mathrm{~Hz}, 2 \mathrm{H}, \mathrm{H}-8 "$ ), 3.34 (s, 3H, $\left.-\mathrm{OCH}_{3}\right), 2.75$ (t, $J=7.8 \mathrm{~Hz}, 2 \mathrm{H}, \mathrm{H}-7$ "); EIMS: m/ $\mathrm{z} 480\left[\mathrm{M}^{+}\right], 465\left[\mathrm{M}-\mathrm{CH}_{3}\right]^{+}, 449\left[\mathrm{M}-\mathrm{OCH}_{3}\right]^{+}, 416\left[\mathrm{M}-\mathrm{SO}_{2}\right]^{+}$, $339\left[\mathrm{M}-\mathrm{PhSO}_{2}\right]^{+}, 141\left[\mathrm{PhSO}_{2}\right]^{+}$.

$N$-3"-Phenylpropyl- $N$-(5-chloro-2-methoxyphenyl)benzene sulfonamide (5k): Colourless gammy solid, Yield: $78 \%$, IR $\left(\mathrm{KBr}, v_{\max }, \mathrm{cm}^{-1}\right): 3015$ (C-H aromatic str.), 1445, $1335\left(\mathrm{SO}_{2}\right), 1224\left(-\mathrm{OCH}_{3}\right), 1155$ (aliphatic C-N str.); ${ }^{1} \mathrm{H}$ NMR (500 MHz, CD 30 OD): $\delta 7.64(\mathrm{dd}, J=7.5,1.5 \mathrm{~Hz}, 2 \mathrm{H}, \mathrm{H}-2$ ' and H-6'), 7.61 (br.t, $J=7.5 \mathrm{~Hz}, 1 \mathrm{H}, \mathrm{H}-4^{\prime}$ ), 7.51 (br.t, $J=7.5 \mathrm{~Hz}$, $2 \mathrm{H}, \mathrm{H}-3^{\prime}$ and $\left.\mathrm{H}-5^{\prime}\right), 7.30$ (dd, $\left.J=8.5,2.5 \mathrm{~Hz}, 1 \mathrm{H}, \mathrm{H}-4\right), 7.19$ (d, $J=2.5 \mathrm{~Hz}, 1 \mathrm{H}, \mathrm{H}-6), 7.16-6.98$ (m, $5 \mathrm{H}, \mathrm{H}-2 "$ to H-6"), $6.91(\mathrm{~d}, J=8.5 \mathrm{~Hz}, 1 \mathrm{H}, \mathrm{H}-3), 3.59$ (t, $\left.J=7.8 \mathrm{~Hz}, 2 \mathrm{H}, \mathrm{H}-9^{\prime \prime}\right)$, $3.36\left(\mathrm{~s}, 3 \mathrm{H},-\mathrm{OCH}_{3}\right), 2.30(\mathrm{t}, J=7.8 \mathrm{~Hz}, 2 \mathrm{H}, \mathrm{H}-7 "), 1.74(\mathrm{~m}$, 2H, H-8"); EIMS: m/z $494\left[\mathrm{M}^{+}\right], 479\left[\mathrm{M}-\mathrm{CH}_{3}\right]^{+}, 463[\mathrm{M}-$ $\left.\mathrm{OCH}_{3}\right]^{+}, 430\left[\mathrm{M}-\mathrm{SO}_{2}\right]^{+}, 353\left[\mathrm{M}-\mathrm{PhSO}_{2}\right]^{+}, 141\left[\mathrm{PhSO}_{2}\right]^{+}$.

\section{RESULTS AND DISCUSSION}

Our objective was to synthesize some new $N$-substituted derivatives of sulfonamides and to screen out their enzymatic activities. Keeping that objective in mind, we synthesized $N$ substitited-(5-chloro-2-methoxyphenyl)benzene sulfonamide in excellent yield and having good biological activities. The synthesis was carried out through the reaction of benzene sulfonyl chloride (1) with 2-amino-4-chloroanisole (2) yielded
$N$-(5-chloro-2-methoxyphenyl)benzene sulfonamide (3) and finally a series of $N$-substituted (5-chloro-2-methoxyphenyl) benzenesulfonamide (5a-k) derivatives were obtained by the reaction of parent compound (3) with different electrophiles (4a-k) in the presence of DMF and sodium hydride $(\mathrm{NaH})$ which act as a strong base represented in Scheme-I. Complete conversion was achieved within 2-4 h by simple stirring at room temperature. The product was isolated by adding cold water in the reaction mixture. It was filtered, washed with distilled water and dried to afford the derivatives (5a-k). The structures of the synthesized compounds were established by ${ }^{1} \mathrm{H}$ NMR (Table-1), IR and mass spectral data as described in experimental section. Compound $\mathbf{3}$ was synthesized as colourless needle like crystals. The molecular formula $\mathrm{C}_{13} \mathrm{H}_{12} \mathrm{NO}_{3} \mathrm{SCl}$ was established by HRMS showing molecular ion peak at $\mathrm{m} / \mathrm{z} 297.7585$ (calcd. for $\mathrm{C}_{13} \mathrm{H}_{12} \mathrm{NO}_{3} \mathrm{SCl}, 297.7582$ ). The IR spectrum revealed the presence of methoxy group $\left(1225 \mathrm{~cm}^{-1}\right)$, a sulfonyl group $\left(1338,1447 \mathrm{~cm}^{-1}\right)$ and -NHgroup $\left(3100 \mathrm{~cm}^{-1}\right)$ in the molecule. The EIMS gave a distinct peak at $\mathrm{m} / \mathrm{z} 233$ after the removal of $-\mathrm{SO}_{2}$ group and further two fragment ion peaks were observed at m/z 282 and 266 which showed the presence of methyl and methoxy groups, respectively in the molecule. In the aromatic region of the ${ }^{1} \mathrm{H}$ NMR spectrum of 3 signals which resonated at $\mathrm{d} 7.41 \mathrm{(d}, J=$ $2.5 \mathrm{~Hz}), 6.79(\mathrm{~d}, J=8.5 \mathrm{~Hz}), 7.05(\mathrm{dd}, J=8.5,2.5 \mathrm{~Hz})$ and $7.41(\mathrm{~d}, J=2.5 \mathrm{~Hz})$ were assigned to the protons of the tri-substituted ring and the signals appeared at $\delta 7.55(\mathrm{t}, J=$ $7.5 \mathrm{~Hz}), 7.45(\mathrm{t}, J=7.5 \mathrm{~Hz})$ and $7.71(\mathrm{dd}, J=7.5,1.5 \mathrm{~Hz})$ were assigned to the protons of the mono-substituted ring. In the aliphatic region of ${ }^{1} \mathrm{H}$ NMR spectrum, a singlet was appeared at $\delta 3.51$ corresponding to the methoxy group present in the molecule. On the basis of these evidences the structure of 3 was assigned as $N$-(5-chloro-2-methoxyphenyl) benzenesulfonamide. The structure of compound $\mathbf{3}$ was also confirmed by single-crystal XRD ${ }^{11,17}$. Similarly on the basis of spectral evidences from IR, EI-MS and ${ }^{1} \mathrm{H}$ NMR, the structures of other derivatives were elucidated as described in experimental section.

Enzyme inhibition activity: The screening of these synthesized compounds against acetylcholinesterase (AChE), butyrylcholinesterase (BChE) and lipoxygenase (LOX) enzymes revealed that these molecules exhibited good inhibitory potential against acetylcholinesterase as it was evident from their $\mathrm{IC}_{50}$ values. It is obvious from Table- 1 that compounds $\mathbf{5 a}, \mathbf{5 e}$ and $\mathbf{5 j}$ were found to be promising inhibitors against acetylcholinesterase enzyme having $\mathrm{IC}_{50}$ value of 34.61 $\pm 0.62,40.21 \pm 0.25$ and $45.11 \pm 0.22 \mu \mathrm{mol} / \mathrm{L}$, respectively, relative to Eserine, a reference standard with $\mathrm{IC}_{50}$ value of 0.04 $\pm 0.001 \mu \mathrm{mol} / \mathrm{L}$, probably due to the $N$-substitution of methyl, allyl and 2-phenylethyl groups, respectively in these molecules. The screening against butyrylcholinesterase enzyme exposed that the compounds $\mathbf{5 a}$ and $\mathbf{5} \mathbf{g}$ exhibited promising inhibitory potential having $\mathrm{IC}_{50} 44.57 \pm 0.04$ and $54.57 \pm 0.05 \mu \mathrm{mol} / \mathrm{L}$ as compared to standard. However, only few compounds (Table1) showed inhibition against lipoxygenase enzyme but all other showed very weak activity. DPPH is a stable free radical at room temperature. DPPH radical is scavenged by antioxidants through the donation of a proton and form reduced DPPH. 
<smiles>Nc1cc(Cl)ccc1O</smiles><smiles>O=S(=O)(Cl)c1ccccc1</smiles>

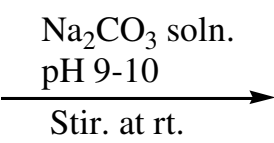

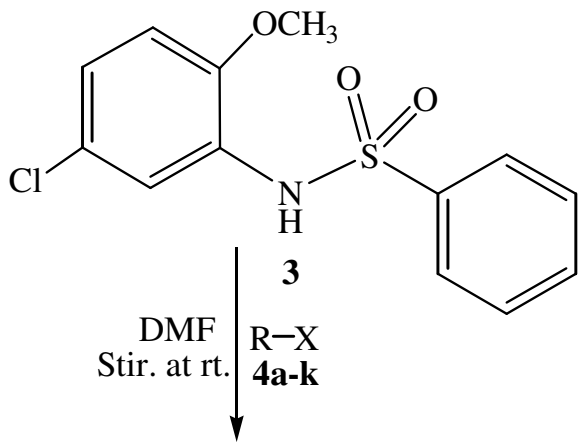<smiles>[R]N(c1cc(Cl)ccc1OC)S(=O)(=O)c1ccccc1</smiles>

5a-k

\begin{tabular}{|c|c|c|c|}
\hline Compound & $\mathrm{R}$ & Compound & $\mathrm{R}$ \\
\hline $5 a$ & $-\mathrm{CH}_{3}$ & $5 \mathrm{~g}$ & $\begin{array}{c}-\mathrm{CH}_{2}-\underset{2^{\prime \prime}}{\mathrm{CH}_{2}}-\underset{3^{\prime \prime}}{\mathrm{CH}_{2}}-\underset{4^{\prime \prime}}{\mathrm{CH}_{2}}-\mathrm{CH}_{3} \\
\end{array}$ \\
\hline $5 b$ & $\begin{array}{l}-\mathrm{CH}_{2}-\mathrm{CH}_{3} \\
1^{\prime \prime}\end{array}$ & $5 \mathrm{~h}$ & 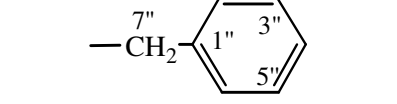 \\
\hline $5 \mathrm{c}$ & $\underset{1^{\prime \prime}}{-\mathrm{CH}}{ }_{\mathrm{CH}_{3}}$ & $5 i$ & 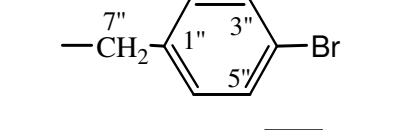 \\
\hline $5 \mathrm{~d}$ & $-\underset{1^{\prime \prime}}{-\mathrm{CH}_{2}-\mathrm{CH}_{2}-\mathrm{Br}}$ & $5 j$ & 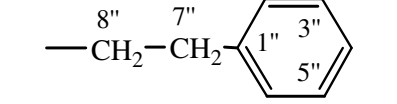 \\
\hline $5 f$ & 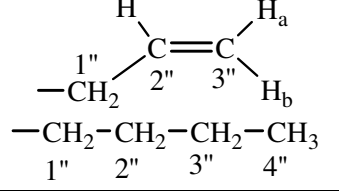 & $5 \mathrm{k}$ & 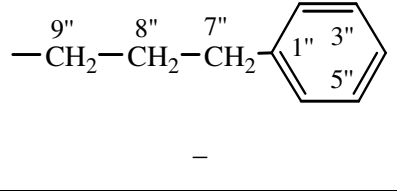 \\
\hline
\end{tabular}

Scheme-I: Outline for synthesis of $N$-substituted (5-chloro-2-methoxyphenyl)benzene sulfonamide

\section{TABLE-1}

BIOACTIVITY STUDIES OF $N$-SUBSTITUTED (5-CHLORO-2-METHOXYPHENYL)BENZENE SULFONAMIDE

\begin{tabular}{|c|c|c|c|c|c|c|c|c|}
\hline \multirow[b]{2}{*}{ C. No. } & \multicolumn{2}{|c|}{ DPPH } & \multicolumn{2}{|c|}{ LOX } & \multicolumn{2}{|c|}{ AChE } & \multicolumn{2}{|c|}{$\mathrm{BChE}$} \\
\hline & $\begin{array}{c}\text { Inhibition (\%) } \\
\text { at } 0.5 \mathrm{mM}\end{array}$ & $\mathrm{IC}_{50}(\mu \mathrm{mol})$ & $\begin{array}{l}\text { Inhibition (\%) } \\
\text { at } 0.5 \mathrm{mM}\end{array}$ & $\mathrm{IC}_{50}(\mu \mathrm{mol})$ & $\begin{array}{l}\text { Inhibition (\%) } \\
\text { at } 0.5 \mathrm{mM}\end{array}$ & $\mathrm{IC}_{50}(\mu \mathrm{mol})$ & $\begin{array}{l}\text { Inhibition (\%) } \\
\text { at } 0.5 \mathrm{mM}\end{array}$ & $\mathrm{IC}_{50}(\mu \mathrm{mol})$ \\
\hline 3 & $89.23 \pm 0.63$ & $98.11 \pm 0.44$ & $50.79 \pm 0.31$ & $398.91 \pm 0.34$ & $63.82 \pm 0.31$ & $139.11 \pm 0.41$ & $58.07 \pm 0.31$ & $295.6 \pm 0.22$ \\
\hline $5 \mathbf{a}$ & $3.63 \pm 0.13$ & Nil & $44.46 \pm 0.41$ & Nil & $87.50 \pm 0.14$ & $34.61 \pm 0.62$ & $25.60 \pm 0.19$ & $44.57 \pm 0.04$ \\
\hline $5 \mathbf{b}$ & $0.57 \pm 0.54$ & Nil & $76.86 \pm 0.34$ & $166.11 \pm 0.24$ & $84.65 \pm 0.22$ & $75.81 \pm 0.04$ & $45.78 \pm 0.45$ & $<400$ \\
\hline $5 c$ & $0.51 \pm 0.22$ & Nil & $64.56 \pm 0.34$ & $<400$ & $74.01 \pm 0.71$ & $132.51 \pm 0.66$ & $64.46 \pm 0.24$ & $180.41 \pm 0.11$ \\
\hline $5 d$ & $0.44 \pm 0.18$ & Nil & $68.94 \pm 0.61$ & $179.11 \pm 0.62$ & $69.85 \pm 0.35$ & $165.61 \pm 0.32$ & $52.08 \pm 0.51$ & $<400$ \\
\hline $5 e$ & $0.76 \pm 0.16$ & Nil & $69.67 \pm 0.13$ & Nil & $69.08 \pm 0.52$ & $40.21 \pm 0.25$ & $67.95 \pm 0.44$ & $<400$ \\
\hline $5 f$ & $89.23 \pm 0.63$ & Nil & $95.13 \pm 0.87$ & $<400$ & $71.93 \pm 0.01$ & $66.71 \pm 0.92$ & $71.72 \pm 0.59$ & $295.6 \pm 0.22$ \\
\hline $5 g$ & $3.63 \pm 0.11$ & Nil & $60.54 \pm 0.25$ & Nil & $54.57 \pm 0.05$ & $213.25 \pm 0.07$ & $39.16 \pm 0.05$ & $54.57 \pm 0.05$ \\
\hline $5 \mathrm{~h}$ & $0.57 \pm 0.54$ & Nil & $56.45 \pm 0.22$ & $384.91 \pm 0.28$ & $57.57 \pm 0.34$ & $192.91 \pm 0.14$ & $37.63 \pm 0.22$ & $57.57 \pm 0.34$ \\
\hline $5 i$ & $0.61 \pm 0.22$ & Nil & $70.16 \pm 0.19$ & $260.81 \pm 0.34$ & $59.10 \pm 0.36$ & $360.81 \pm 0.25$ & $60.09 \pm 0.11$ & $59.10 \pm 0.36$ \\
\hline $5 \mathbf{j}$ & $0.56 \pm 0.12$ & Nil & $47.50 \pm 0.09$ & Nil & $78.73 \pm 0.59$ & $45.11 \pm 0.22$ & $92.84 \pm 0.09$ & $<400$ \\
\hline $5 k$ & $0.58 \pm 0.15$ & Nil & $77.71 \pm 0.59$ & $144.43 \pm 0.18$ & $66.12 \pm 0.73$ & $67.91 \pm 0.41$ & $63.82 \pm 0.59$ & $66.12 \pm 0.73$ \\
\hline Control & $\begin{array}{c}\text { Quercetin } \\
93.21 \pm 0.97\end{array}$ & $16.96 \pm 0.14$ & $\begin{array}{c}\text { Baicalein } \\
93.79 \pm 1.27\end{array}$ & $22.4 \pm 1.3$ & $\begin{array}{c}\text { Eserine } \\
91.29 \pm 1.17\end{array}$ & $0.04 \pm 0.001$ & $\begin{array}{c}\text { Eserine } \\
82.82 \pm 1.09\end{array}$ & $0.85 \pm 0.001$ \\
\hline
\end{tabular}

Note: $\mathrm{IC}_{50}$ values (concentration at which there is $50 \%$ enzyme inhibition) of compounds were calculated using EZ: Fit enzyme kinetics software (Perella Scientific Inc. Amherst, USA). LOX = Lipoxygenase. AChE = Acetyl cholinesterase. $\mathrm{BChE}=\mathrm{Butyryl}$ cholinesterase. $\mathrm{DPPH}=$ 1,1-diphenyl-2-picrylhydrazyl radical. 
The colour changes from violet to yellow after reduction of DPPH and it can be quantified by decrease of absorbance at wavelength $517 \mathrm{~nm}$. Radical scavenging activity increased with increasing percentage of the free radical inhibition. The colour change from violet to yellow and fall in absorbance of the stable radical DPPH was measured for three different concentrations of samples and the results are shown in Table- 1 . These results showed that the parent compound $N$-(5-chloro-2methoxyphenyl)benzene sulfonamide (3) showed scavenging activity against 1,1-diphenyl-2-picrylhydrazyl (DPPH) radical but all others remained inactive. The $\mathrm{IC}_{50}$ value for the parent compound $98.11 \pm 0.44 \mu \mathrm{mol} / \mathrm{L}$, relative to quercetin, a reference standard with $\mathrm{IC}_{50}$ value of $16.96 \pm 0.14 \mu \mathrm{mol} / \mathrm{L}$, was calculated from the curves plotted. $\mathrm{IC}_{50}$ is the concentration of fraction causing $50 \%$ inhibition of absorbance and lower its value means greater antioxidant activity of the fraction.

\section{Conclusion}

The proposed structure of the synthesized compound is well supported by spectroscopic data. From the enzyme inhibition data (Table-1), it might be concluded that the three compounds $\mathbf{5 a}, \mathbf{5 e}$ and $\mathbf{5 j}$ have promising activity against acetylcholinesterase enzyme as compared to relative standard. Some synthesized compounds exposed moderate activity against butyrylcholinesterase and lipoxygenase enzymes but only parent compound $\mathbf{3}$ showed scavenging activity against DPPH but all were remained inactive. Hence on the basis of aforesaid results, these synthesized derivatives provide an overall indispensable basis to introduce new drug candidates for the treatment of Alzheimer's disease and other associated diseases.

\section{REFERENCES}

1. C.T. Supura, A. Scozzafava, L. Menabuoni, F. Mincione, F. Briganti and G. Mincione, Eur. J. Pharm. Sci., 8, 317 (1999).

2. M. Remko and C.W.V. Lieth, Bioorg. Med. Chem., 12, 5395 (2004).

3. G.L. Perlovich, N.N. Strakhova, V.P. Kazachenko, T.V. Volkova, V.V. Tkacher, K.J. Schaper and O.A. Raevsky, Int. J. Pharm., 349, 300 (2008).

4. A.K. Gaded, C.S. Mahajanshetti, S. Nimbalkar and A. Raichurkar, Eur. J. Pharm. Sci., 35, 853 (2000).

5. N.S. El-Sayed, R.E. El-Bendary, S.M. El-Ashry and M.M. El-Kerdawy, Eur. J. Pharm. Sci., 46, 3714 (2011).

6. M.J. Garcia-Galan, M.S. Diaz-Cruz and D. Bercelo, Trend. Anal. Chem., 27, 1008 (2008).

7. M. Cygler, J.D. Schrag, J. Sussman, L.M. Harel, I. Silman and M.K. Gentry, Protein Sci., 2, 366 (1993).

8. V. Tougu, Curr. Med. Chem., 1, 155 (2001).

9. S. Gauthier, Drugs Aging, 18, 853 (2001).

10. G. Bertaddini, Handbook of Experimental Pharmacology, Springer, Berlin, 59/II, 85 (1982).

11. Aziz-ur-Rehman, W. Tanveer, M.A. Abbasi, S. Afroz, K.M. Khan, M. Ashraf and I. Afzal, Int. J. Chem. Res., 3, 99 (2011).

12. G.L. Ellman, K.D. Courtney, V. Andres and R.M. Featherstone, Biochem. Pharmaco., 7, 88 (1961).

13. A.L. Tappel, Arch. Biochem. Biophys., 44, 378 (1953).

14. A.T. Evans, Biochem. Pharmaco., 36, 2035 (1987).

15. S. Baylac and P. Racine, Int. J. Aromatherap., 13, 138 (2003).

16. V. Koleva, T.A. Beek, J.P.H. Linssen, A. de Groot and L.N. Evstatieva, Phytochem. Anal., 13, 8 (2002).

17. Aziz-ur-Rehman, Shahzaman, M. Akkurt, M.A. Abbasi and I.U. Khan, Acta Cryst., E66, o2855 (2010). 